\title{
Time Dependent Conductivity in Disordered Systems
}

\author{
G. F. Leal Ferreira and S. C. Costa \\ Instituto de Física de São Carlos - Universidade de São Paulo \\ C.P. 369, 13560-970, São Carlos, SP, Brazil
}

Received 18 February, 1998; received in revised form 3 August, 1998

\begin{abstract}
The time dependent current in disordered systems under a step applied voltage for a planar symmetry is deduced according to the continuous time random walk approximation. Known dielectric response functions like Cole-Cole, Davidson-Cole, Havriliak-Negami and a few others are used as hopping time distribution functions in order to generate conductive responses. A theoretical relation exists between the dielectric and the conductive response which is the same one prevailing between the time derivative of the creep and the relaxation function, as found long ago by Gross (J.Appl.Phys.,18, 212 (1947)). A truncated version of the Widder method, in connection with MapleTM software facilities, was employed to obtain graphical primitives of Laplace transforms.
\end{abstract}

\section{Introduction}

In the opening talk "Electret Research - Stages in its Development" [1] of the 5th International Symposium on Electret, ISE 5, held at Heidelberg, honoring his 80th birthday, Prof Gross reviewed the electret research at its very early stages. This testimony is particularly relevant on account of the role Prof Gross played in the development of the field. He succeeded experimentally in distinguishing between hetero and homocharges appearing in poled dielectrics [2]. Heterocharge is the charge with opposite polarity to that residing on the nearby electrode, while the homocharge has the same sign. The former is attributed to polarization and the latter to space charge injection. Polarization in most cases is a linear effect while space charge is not. See also [3], and for the latest developments in this topic, see [4].

In dielectrics, polarization obeys a superposition principle $[1,5]$, by which the material presents a delayed response to changes of the poling electric field. The simplest response of the polarization to a step variation in the electric field is characterized by an exponential increase (the so called Debye response), but this is rarely observed experimentally. Anyway the delayed response of the polarization to an alternating applied voltage gives rise to dielectric losses. In this kind of measurement the real and the imaginary components are related through the Kramers-Kronig [3] relations. To these relations Prof. Gross arrived working independently $[6]$.
Here we will be concerned with a new type of delayed response: that afforded by current flowing in disordered materials. That is, absorption currents appear not only due to dipoles during the localized orientation process but also to the unbound motion of a drifting carrier in a disordered medium.

The theoretical study of transport in a disordered medium was considerably advanced in a series of works which culminated with the famous article by Scher and Montroll (SM ) [7-10], using the Continuous Time Random Walk approach (CTRW). In this treatment, the carrier performs a random walk in a regular lattice, biased by an electric field, and the disordered character of the medium reflects itself in the hopping time distribution function (HTDF), which now deviates from a simple exponential. For time dependent electric fields the SM approach leads to a result [11] that does not agree with the Kubo fluctuation theorem as mentioned by Dyre [12]. This latter author then introduced a correction within the CTRW approach [12] and obtained results which were shown to be close to the effective medium alternative treatment (despite previous criticism on the accuracy of the CTRW approximation , see [12], section $V$ and [13]). We stick to the CTRW in the following. A phenomenological attack to the problem has been carried out by J.R.Macdonald [14-16] and in [17]. See also $[18,19]$.

In the CTRW description, the important quantity is the HTDF, a decaying function of the time, which is the negative of the time derivative for the probability 
of the carrier to remain in the same site for some time after its arrival in it. The effect of disorder is to introduce hereditariness which makes the current to depend on past values of the electric field through a function related to the HTDF. This is very akin to the dielectric case in which the HTDF, as a waiting time distribution function for localized motions, is itself the hereditary function. Since dielectric theory has produced a bunch of such functions which reflect the prevailing order-disorder, it seems worthwhile to consider them as possible HTDF for conductive processes. Therefore Cole-Cole, Davidson-Cole, Havriliak-Negami, and a few other distributions will be used for obtaining conductive responses. In this aspect our approach is phenomenological.

We start from Dyre's analysis of the CTRW approximation [12] presented in a somewhat different manner [20], as shown next.

\section{Theoretical background}

The SM result for the current density, $i_{c}(x, t)$, due to the space charge density $\rho(x, t)$, moving in a constant electric field $E_{0}$ in a planar symmetry is

$$
i_{c}(x, t)=a E_{0} \int_{0}^{t} \phi\left(t-t^{\prime}\right) \rho\left(x, t^{\prime}\right) d t^{\prime}
$$

where $\phi(t)$ is a function related to the HTDF, $\Psi(t)$, as will be given shortly. $\underline{a}$ is a constant with dimension of mobility/time. A generalization of Eq.1 to time (and space) dependent electric fields [11] maintains the electric field outside the integral and this makes the result to disagree with Kubo's current-current fluctuation theorem, [12], for which fluxes correlate in time. The main result of Dyre's analysis, which was originally devoted to ac processes (see Eq.17 of [12] and the paragraph below Eq. 8 in the following), is, in the time domain, to transfer the electric field inside the integral in Eq.1. If additionally the mobile space charge density in a compensate system is assumed to be constant - as if the material was endowed with a conductivity - Eq.1 becomes

$$
i_{c}(t)=A \int_{0}^{t} \phi\left(t-t^{\prime}\right) E\left(t^{\prime}\right) d t^{\prime}
$$

A in Eq.2 is a constant with the dimension of conductivity/time. If the transport happens in a non-disordered medium, $\phi(t)$ is the product of a frequency $w$ and a delta function and Eq.2 becomes

$$
i_{c}(t)=A w E(t)
$$

with the product Aw defining the conductivity $\sigma$. For hopping transport $\sigma$ is [21]

$$
\sigma=e n w d
$$

where $e$ is the electronic charge, $n$ is the density of mobile charges, and $d$ the mean hopping distance for a unit electric field.

The function $\phi(t)$ is related to the HTDF, $\Psi(t)$, in the Laplace space, through the following relation [10]

$$
\phi^{*}(s)=\frac{s \Psi^{*}(s)}{1-\Psi^{*}(s)}
$$

where $\phi^{*}(s)$ and $\Psi^{*}(s)$ mean the Laplace Transforms (LT) of $\phi(t)$ and $\Psi(t)$, respectively. That is, for instance

$$
\Psi^{*}(s)=\int_{0}^{\infty} e^{-s t} \Psi(t) d t
$$

For a given $\Psi(t), \phi(t)$ will be known after inversion of Eq.5.

The HTDF is related to the probability for the carrier to remain for a time $t$ after arriving at a site, $\Phi(t)$, through

$$
\Psi(t)=-\frac{d \Phi}{d t}
$$

or in the Laplace space

$$
\Psi^{*}(s)=1-s \Phi^{*}(s)
$$

In Eq. 8 we have used the condition $\Phi(0)=1$. If the medium is ordered, $\Phi(t)$ becomes an exponential, viz. $e^{-w t}$, and after inversion, $\phi(t)$ comes out as $w \delta(t)$ as already asserted. Eq.2 tells us that the conduction current is exactly in phase with the applied field. To make a connection with the Dyre work, it is he L.T. of $\Phi(t)$ that corresponds to the mean appearing in Eq.17 of [12] for the ac case, with $s \rightarrow i \omega, \omega$ being the angular ac frequency.

The merit of the Dyres analysis is to provide a single formalism for treating $d c$ as well as ac responses as they both follow from Eq.2. See [22] for a different view of this topic.

\section{The delayed response of conductive systems}

\section{III.1 Generating conductive responses}

The delayed current density response of a pure dielectric (that is, without a conductivity), $i_{d}(t)$, has $\Psi(t)$ 
itself, defined in Eq.7, as the hereditary function. But the process is now localized and $\Psi(t)$ becomes the waiting time distribution function of the process

$$
i_{d}(t)=C \int_{0}^{t} \Psi\left(t-t^{\prime}\right) \frac{d E\left(t^{\prime}\right)}{d t^{\prime}} d t^{\prime}
$$

where $C$ is a constant with the dimension of conductivity/time. In a microscopic model $\Psi(t)$ results either from a distribution of relaxation times (here, against tradition, relaxation frequencies will be used instead) or from non-Debye processes (see the end of section 34). According to Eq.9, the current density depends on the superposed effects of all the past variations of the electric field, $E(t)$. In Eq.9 we take $E(0)=0$. The generating function $\Psi(t)$ will be called the dielectric response function (DRF) when referring to a dielectric response. But it may also generate formally a conductive response as a HTDF, see Eqs. 2 and 5. The conductive current density response, $i_{c}(t)$, would then be found using Eq.2, after an adequate inversion of Eq.5. In order to find a theoretical relationship between dielectric and conductive responses we now consider Eqs.2 and 9. We note first that Eq.2 and Eq.9 are differently related to the electric field. In order to put the two response functions on the same footing we will change Eq.2 to look like Eq.9. We therefore write $i_{c}(t)$ as

$$
i_{c}(t)=A \int_{0}^{t} F\left(t-t^{\prime}\right) \frac{d E\left(t^{\prime}\right)}{d t^{\prime}} d t^{\prime}
$$

and endeavor to find $F(t)$. For this let us carry a LT of both equations, Eq.2 and 10.

$$
\phi^{*} E^{*}=s F^{*} E^{*} .
$$

Therefore we get

$$
s F^{*}=\phi^{*}
$$

or finally

$$
F^{*}(s)=\frac{\phi^{*}(s)}{s}
$$

Let us digress on this result. If we put

$$
\left(\frac{d F(t)}{d t}\right)^{*}=s F^{*}-F(0)
$$

we conclude from Eqs.12 and 14 that

$$
\frac{d F(t)}{d t}=\phi(t)-F(0) \delta(t) .
$$

This equation indicates that $\phi(t)$ always incorporates a delta function. Indeed, using Eq.5 and finding the limit for $s \rightarrow \infty$ of $s \phi^{*}$ we get

$$
\phi(0)=\lim _{s \rightarrow \infty} s \phi^{*}(s)=\lim _{s \rightarrow \infty} s \frac{s \Psi^{*}(s)}{1-\Psi^{*}(s)}=\Psi(0) s \rightarrow \infty
$$

since the limit of $s \Psi^{*}(s)$ for $s \rightarrow \infty$ is $\Psi(0)$, which is finite for well behaved $\Psi(t)$; additionally $\lim _{s \rightarrow \infty} \Psi^{*}(s) \rightarrow 0$ and Eq.15 follows. In Section IV-2 we shall show that $F(0)=\Psi(0)$. Eq.14 then means that the time derivative of $F(t)$ equals the regular part of the function $\phi(t)$.

Returning to Eq.13 and writing $F^{*}(s)$ in terms of $\Psi^{*}(s)$ using Eq.5, we get

$$
F^{*}(s)=\frac{\Psi^{*}(s)}{1-\Psi^{*}(s)}
$$

$F(t)$ will be called the conductive response function (CRF), or else, the conductivity ignoring for short the constant factor $A$ in Eq.10. It is also convenient to separate from $F(t)$ the part containing the ultimate conductivity $\sigma$. The LT of the resulting function, $F_{t}^{*}(s)$, is

$$
F_{t}^{*}(s)=\frac{\Psi^{*}(s)}{1-\Psi^{*}(s)}-\frac{\sigma}{s}
$$

with $\sigma=\Psi^{*}(0) /\left(1-\Psi^{*}(0)\right)$. Fig.1 illustrates the contributions of $F(t), F_{t}(t)$ and for unit electric field applied at $t=0$ and for a long time, followed by a short circuit from $t_{0}$. In Eq.10 there will appear two delta functions in the time derivative of the field, one for $t=0$, positive and the other, negative, for $t=t_{0}$, due to the two steps in the electric field (see Fig.1). Therefore, besides the initial decaying current, a return current appears for $t \geq t_{0}$, as in the dielectric case. For sufficient large $t_{0}$ the returning current is $-F_{t}\left(t-t_{0}\right)$. We observe that the superposition of a independent conduction mechanism on the pure dielectric response, Eq.9, makes it difficult to distinguish between DRF with a conductivity and a CRF.

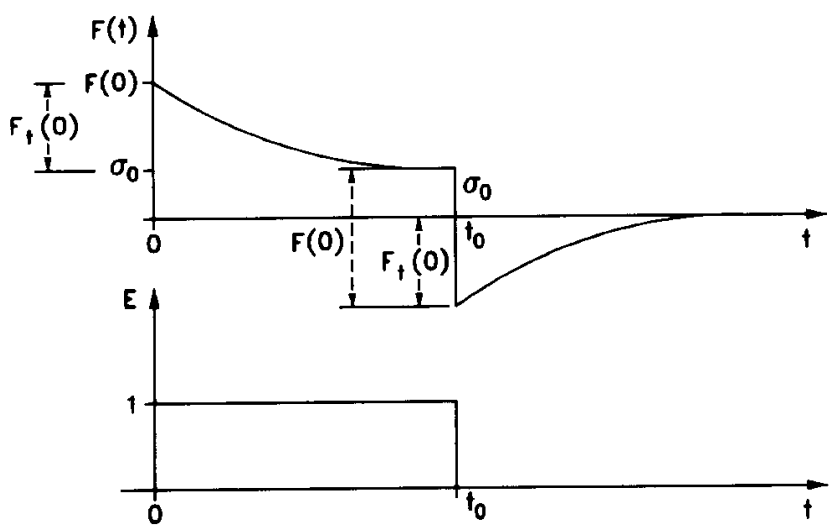

Figure 1. Schematic representation of the conductive current (upper) following the application of a unit electric field 
at $t=0$ and its discontinuation after a long time $t=t_{0}$, according to the Superposition Principle. For the meaning of the symbols see the text. Note the return current appearing after $t_{0}$.

A direct comparison between a DRF and CRF derived from a single HTDF by means of Eq.17 does not seem realistic inasmuch as the parameters by means of which they are determined are not expected to be the same (referring to localized and non-localized motion, respectively). In particular we expect the return current in Fig. 1 to be of short duration when compared with the dielectric ones, and therefore the same must be true for the corresponding absorption currents. But we carry on comparing DRF and CRF in order to make connection with the work of Prof. Gross in Viscoelasticity and with other areas in which a similar relationship between two related functions appears. The reader not interested on these analogies should skip the next section, returning to the text below Eq.25.

\section{III.3 Analogy with pure dielectric re- sponses}

Viscoelastic effects are accessed through two kinds of experiments, creep and relaxation $[5,23,24]$. In the former the material creeps under constant force while in the latter the force relaxes under constant creep. The obtained creep and relaxation functions are interrelated through their LTs, as shown in [23]. In dielectric theory a single function, $\Psi(t)$, is in use but it is easy to create its counterpart as in viscoelasticity. For this we recognize that what Maxwell (properly) called the displacement, $D(t)$, is the analogue of creep while the electric field, $E(t)$, is the analogue of force. We then write, as for the viscoelastic case [23], the equations based on the Superposition Principle

$$
D(t)=\epsilon\left[E(t)+\int K_{E}\left(t-t^{\prime}\right) \frac{d E}{d t^{\prime}} d t^{\prime}\right]
$$

and

$$
E(t)=\frac{1}{\epsilon}\left[D(t)-\int K_{D}\left(t-t^{\prime}\right) \frac{d D}{d t^{\prime}} d t^{\prime}\right]
$$

where $K_{E}(t)$ and $K_{D}(t)$ define the retarded response for changing electric field and for changing displacement, respectively. Note the positive sign in front of the integral in Eq.19 in contrast to the negative one appearing in Eq.20.

Direct access to $K_{D}(t)$ is possible through experiments with a constant current source or a constant current triode [25-27]. Fig.2 shows how to obtain $K_{D}(t)$. On the other hand it is not difficult to see that the negative of the time derivative of $K_{E}(t)$ is just $\Psi(t)$, Eq.9.
Then operating with Eqs.19 and 20 in the Laplace space we find that

$$
\Psi^{*}=\left(-\frac{d K_{E}}{d t}\right)^{*}=\frac{s K_{D}^{*}}{1-s K_{D}^{*}}
$$

Fig. 2 shows that $K_{D}(0)=0$ and therefore $s K_{D}^{*}$ is the LT of $d K_{D} / d t$. Going back to Eq.17 we see that $s K_{D}^{*}$ is to $\Psi^{*}$ as $\Psi^{*}$ is to $F^{*}$. It may be seen that if $\Psi$ is an exponential like $w e^{-w t}, d K_{D} / d t$ is also an exponential but going as $w e^{-2 w t}$.

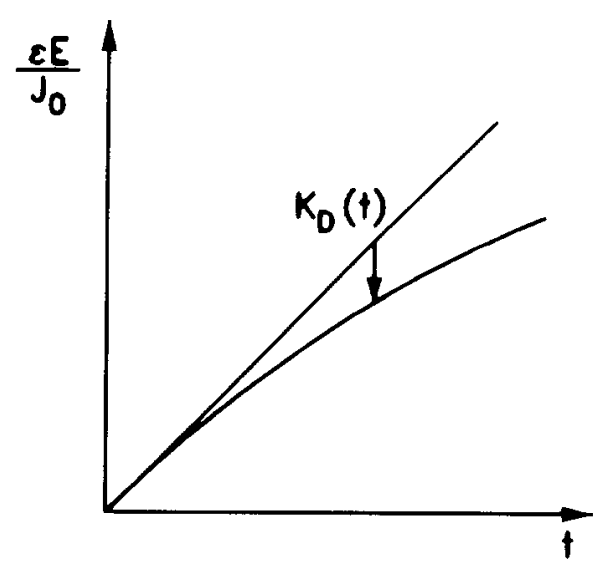

Figure 2. Experimental determination of the retarded response $K_{D}(t)$, Eq.20, by means of source of constant current $J_{0}$. is the high frequency dielectric constant.

\section{III.4 Relation between the spectra of $\Psi$ and $F$}

The function $\Psi(t)$ is a decaying function of time and therefore may be considered the LT of a positive function, $g_{\Psi}(w)$, of the relaxation frequency, or, for short, of the frequency $w$ [28]

$$
\Psi(t)=\int_{0}^{\infty} e^{-w t} g_{\Psi}(w) d w
$$

Eq.22 shows that $d \Psi / d t$ is indeed negative for positive $g_{\Psi}(w)$.

On physical grounds let us assume that the CRFs are also decaying functions of time, or at least nonincreasing ones. Therefore they may also be expressed as the LT of a positive spectrum $g_{F}(w)$,

$$
F(t)=\int_{0}^{\infty} e^{-w t} g_{F}(w) d w
$$

For $\Psi(t)=w^{\prime} e^{-w^{\prime} t}, g_{F}(w)$ turns out to be $w^{\prime} \delta(w)$, giving rise to a pure conductive response.

We may profit now from the previous work of Prof. Gross on Viscoelasticity $[29,23]$ in order to derive a relation between $g_{\Psi}(w)$ and $g_{F}(w)$. It is found that

$$
g_{F}(w)=\frac{g_{\Psi}(w)}{\left(1-P_{\Psi}(w)\right)^{2}+\pi^{2} g_{\Psi}^{2}(w)}
$$


where $P_{\Psi}(w)$ is the Cauchy principal value of the integral [29]

$$
P_{\Psi}(w)=\int_{0}^{\infty} \frac{g_{\Psi}(\eta) d \eta}{\eta-w}
$$

By Eq.24, $g_{F}(w)$ is also positive and $F(t)$ is indeed a non-increasing function of the time.

The frequency spectrum may contain lines in which case $g_{\Psi}$ will be a discrete sum containing one or more delta functions. As we expect Eq.24 to remain formally valid, squares and products of delta functions will appear; this aspect was explored by Gross and Pelzer [31] and Gross [32,33]. In a private conversation with one of us (GFLF) Prof. Gross expressed the opinion that insufficient attention has been given to this subject by the mathematically skilled community.

As we have seen the assignment of a spectrum to DRF and to CRF raises interesting mathematical problems. Whether such distributions have an ultimate physical reality - that is whether they may be justified microscopically -, is a different question (see introductory remarks in [33], by Prof. Gross). Some authors suggest that non-Debye processes exist on their own [9,35-38] while others think the opposite [14-16,11,39]. Williams and Watts [40] assign a distribution for the $\beta$ relaxation in polymers having dipoles attached to side groups and attribute a non-Debye process for the $\alpha$ relaxation occurring at the glass transition temperature. For our formal approach the question is not relevant.

\section{III.5 The amplitude of the CRF}

A point of interest is to compare the amplitude of $F_{c}(t)$ with that of $F(t)$, at $t=0$, the latter including the dc component according to Eq.17. First, it may be shown that $F_{c}(0)=\Psi(0)$. Indeed we have

$$
\lim _{s \rightarrow \infty} s \Psi^{*}(s)=\Psi(0) \text { and } \quad \lim _{s \rightarrow \infty} s F_{c}(s)=F_{c}(0)
$$

Therefore

$$
F_{c}(0)=\lim _{s \rightarrow \infty} s \Psi_{c}^{*}(s)=\lim _{s \rightarrow \infty} \frac{s F^{*}(s)}{1-\Psi^{*}(s)}=\Psi(0)
$$

since $\Psi^{*}(\infty)=0$, according to the well known property of the LT.

On the other hand, when $t \rightarrow \infty$, we have $\Psi(\infty)=0$ while $F_{c}(\infty)$ tends to $\sigma$, Eq.18. Therefore, we conclude that the CRF, $F_{c}(t)$, decays less than the DRF, $\Psi(t)$ which generates it. This is in accord with our intuition in that we expect a stronger decay in a dielectric than in a conductive system. A few examples will be shown later.

\section{Obtaining CRF from its generating DRF}

\section{IV.1 System with two line frequencies}

As an introduction to the study of CRFs derived from distributions, let us suppose that the generating DRF has two frequencies, $w_{1}$ and $w_{2}$, with probabilities $a$ and $1-a$. That is, $\Psi(t)$ is given by

$$
\Psi(t)=a_{1} w_{1} e^{-w_{1} t}+(1-a) w_{2} e^{-w_{2} t}
$$

As a HTDF it generates the CRF, $F(t)$, which after a somewhat lengthy inversion of Eq.17 gives

$$
F(t)=\frac{a(1-a)\left(w_{1}-w_{2}\right)^{2}}{w_{1}(1-a)+a w_{2}} e^{-\left[w_{1}(1-a)+w_{2} a\right] t}+\frac{w_{1} w_{2}}{w_{1}(1-a)+a w_{2}}
$$

It is interesting to interpret this result. If we assign to two resistors, $R_{1}$ and $R_{2}$, resistance values proportional to $1 / w_{1}$ and $1 / w_{2}$, respectively, the conductance at $t=0, R^{-1}(0)$, is given by the weighted sum of the two resistors in parallel

$$
R^{-1}(0)=\frac{a}{R_{1}}+\frac{1-a}{R_{2}} \propto a w_{1}+(1-a) w_{2}
$$

as may be verified from Eq.29. On the other hand, for $t \rightarrow \infty$, the resistance $R(\infty)$ results from the weighted combination of the resistors in series

$$
R(\infty)=a R_{1}+(1-a) R_{2} \propto \frac{a}{w_{1}}+\frac{1-a}{w_{2}}
$$

The combinations resulting in $R(0)$ and $R(\infty)$ are shown in Fig.3. We then see that if $R_{2}$ is much greater than $R_{1}$ and $a \approx 1 / 2$, the fastest routes prevail for short 
times, $\left(R^{-1}(0) \approx 1 / 2 R_{1}\right)$ while the slowest prevail for long times $\left(R(\infty)=R_{2} / 2\right)$. a)

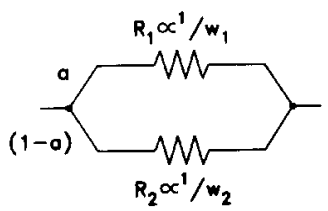

b)

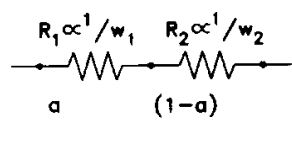

Figure 3. a) weighted parallel combination of resistors, $R_{1} \propto \leq 1 / w_{1}$ and $R_{2} \propto 1 / w_{2}$, giving the initial conductance; b) weighted series combination of resistors giving the dc conductivity.

\section{IV.2 Highly dispersive response}

A CRF deprived of a dc component will be called highly dispersive (HDCRF). A HTDF with an asymptotic hyperbolic behavior is a common ingredient for obtaining such responses. Let us denote it, as usual, as $\Psi(t)$. When the asymptotic behavior is hyperbolic $\Psi^{*}(s)$ for $s \rightarrow 0$, or $t \rightarrow \infty$, is expressed as

$$
\lim _{s \rightarrow 0} s \Psi^{*}(s) \approx s\left(1-s^{m}\right), \text { for } 0 \leq m \leq 1
$$

from which the following temporal behavior derives $[23,41]$

$$
\Psi(t)_{t \rightarrow \infty} \approx-\frac{t^{-m-1}}{,(-m)}
$$

where,$(-m)$ is the gamma function of the negative argument obeying [42]

$$
,(-m),(m)=-\frac{\pi}{m \sin \pi m}
$$

This concerns the DRF. For the CRF, the asymptotic behavior is readily found with Eqs.17 and 32

$$
\lim _{s \rightarrow 0} s F_{c}(s)=\lim _{s \rightarrow 0} \frac{s \Psi^{*}(s)}{1-\Psi^{*}(s)}=\frac{s\left(1-s^{m}\right)}{s^{m}}=s^{1-m}-s
$$

According to well known rules $[23,43,44]$ this gives

$$
F(t)_{t \rightarrow \infty} \approx \frac{t^{-(1-m)}}{,(m)}
$$

Let us compare the two asymptotic behaviors, Eqs.33 and 36 . We see that DRF decays more steepely, with exponent $-(m+1)$, while the CRF decays with an exponent $-(\mathrm{m}-1)$. The exponents add to -2 , a kind of universality similar to the one obtained by SM [10] for current traces in certain disordered materials excited by a light pulse. As already commented upon the universality found here between DRF and CRF is at most of mathematical interest.

\section{IV.3 Normal dispersive conductive re- sponses}

In the previous sub-section we have treated the highly dispersive CRF. Normal dispersive CRFs (ND$\mathrm{CRF}$ ) are those showing relaxation as well as a dc conductivity for $t \rightarrow \infty$. The two barrier system of subsection IV.3, the Davidson-Cole generated CRF and the random free energy barriers of Macdonald [14] and Dyre [12] exemplify this case (see later). Stretchedexponentials also give rise to normal dispersive responses

\section{IV.4- The inversion of the LT}

Whenever possible we have used tables [43,44] for handling inverse LTs. When numerical analysis became necessary, we have employed a truncated version of Widder's method [45], in which the primitive $f(t)$ is obtained from its LT $f^{*}(s)$ through the algorithm

$$
f_{k}(t)=\frac{(-)^{k}}{k !}\left(\frac{k}{t}\right)^{k+1} f^{*(k)}\left(\frac{k}{t}\right)
$$

in the limit $k \rightarrow \infty$. The superscript $(k)$ on $f^{*}$ indicates the $k^{t h}$ order derivative. We have found that in many cases a relatively low $k$ makes the graphical representation of $g_{k}(t)$ and of $g_{k+1}(t)$ to coincide under visual inspection. We took this to mean that a sufficiently good approximation was achieved. In he calculations the $5^{\text {th }}$ version of the Maple ${ }^{T M}$ software facilities was used. In the following a few examples of the use of this truncated Widder method are given. Results with the LT $1 / \sqrt{s}$, whose primitive is $1 / \sqrt{\pi t}$, are shown in Fig.4 for $k=2,3$, and the exact one. In Fig.5, we present the inversion of $1 /\left(1+s^{3 / 4}\right)$, with no available primitive, for $k=2,6$ and 10, the last one being already the correct solution according to visual inspection. In Fig.6 the inversion of $e^{-\sqrt{s}}$ is shown for $k=30$ and 50 as well as the exact solution $\left(e^{-1 /(4 t)} / 2 \sqrt{\pi t^{3}}\right)$. We see that the convergence becomes slower near the maximum and therefore it seems that the method is better suited to functions whose primitives are decaying functions of the time, such as DRF and CRF. Hyperbolic decay makes convergence easier. See also Fig.12 later. 


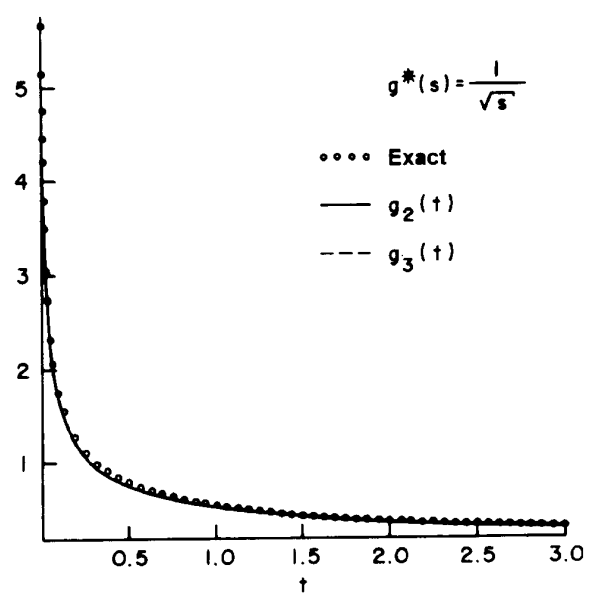

Figure 4. Approximated primitives of $1 / \sqrt{s}$ using truncated Widder's formula, Eq.37, for $k=2$ and 3, and the exact primitive. The $k=3$ is scarcely seen between the $k=2$ curve and the exact result.

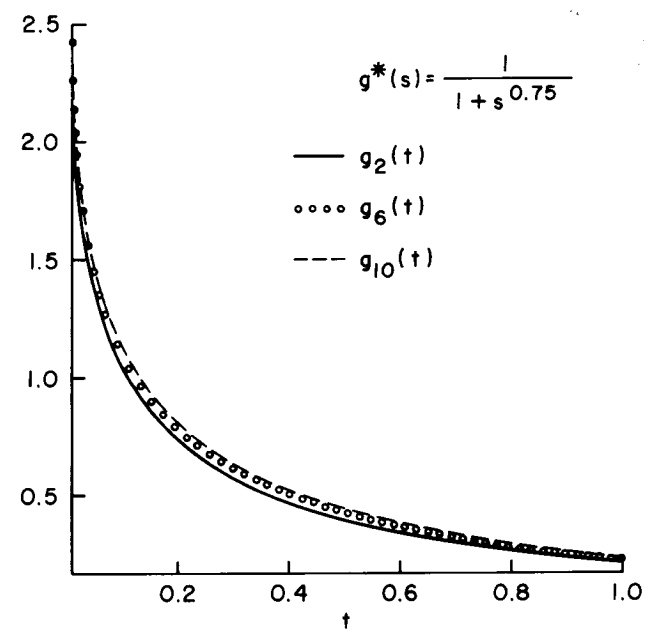

Figure 5. Approximated primitives of $1 /\left(1+s^{3 / 4}\right)$ for $k=2$, 6 and 10, using the truncated Widder method. The last one may be considered visually exact.

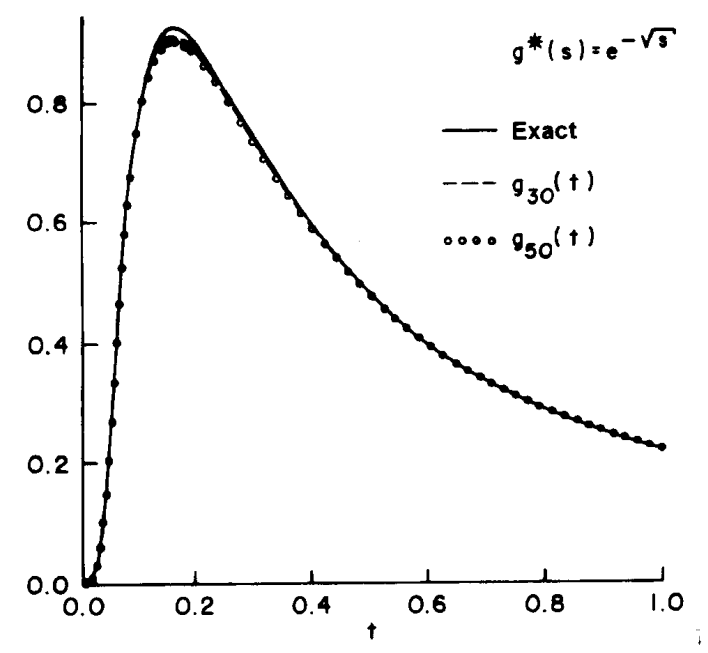

Figure 6. Approximated primitives of $e^{-\sqrt{s}}$, using Eq.37, for $k=30,50$ and the analytical primitive.

\section{Derivation of CRFs in spe- cific cases}

\section{V.1 Preparation}

In the following we obtain CRFs, from DRFs acting as HTDFs, with the use of Eq.17. Most of the DRFs originated from ac measurements and were inferred from attempts to fit results plotted in a ColeCole diagram $[3,19,46]$, involving the real and the imaginary part of the complex susceptibility. By means of either Eq.9 [49] or through generalizations performed upon the pure Debye ac response, it may be shown that the complex susceptibility is proportional to the LT (through a factor $C$ in Eq.9) of the DRF when making $s \rightarrow i \omega$, with $i=\sqrt{-1}$ and $\omega$ being the angular frequency. For instance, the Cole-Cole complex susceptibility derives from

$$
\Psi_{C C}^{*}(i \omega)=\frac{1}{1+(i \omega)^{1-\alpha}} \quad, \quad 0 \leq \alpha \leq 1
$$

and the DRF is just $\Psi_{C C}^{*}(i \omega \rightarrow s)$. Therefore, the task of finding the CRF through Eq.17 is greatly simplified for the other dielectric functions having similar origin (like the Davidson- Cole and the Havriliak-Negami functions; this is not the case with Jonscher's Universal response $[18,35,36]$, which for this reason will not be considered here).

Some care is necessary when analyzing in the time domain a derived CRF. For instance, it is easily seen that $s \Psi_{C C}^{*}(s)$ in Eq.38 diverges for $s \rightarrow \infty$. Therefore, $\Psi_{C C}(t \rightarrow 0) \rightarrow \infty$ and this shows that the Cole-Cole DRF works only for sufficiently large $t$. However this time is in many cases small in relation to the experimental time scale employed, and therefore we will take such responses as meaningful over essentially the whole time scale. The same may be said of the stretchedexponentials, as explained below.

While presenting the results we keep the nomenclature of time dependent conductivities when referring to $F$ or $F_{t}$. Of course true conductivities include a factor $\mathrm{A}$ as in Eq.10.

\section{V.2 Specific highly dispersive responses}

The CRF generated by the common dielectric responses will be considered. Starting with the HDCRF case, we will consider the Cole-Cole 
DRFs $[3,19,24,39,46,48]$, which constitute a oneparameter family of functions, and the HavriliakNegami $[19,46,48,49]$ ones, which is a two parameter distribution function. For the latter, no analytical result could be derived [49] and so only the short and the long time behavior are given. For the former it so happens that a very simple expression results for the HDCRF. These distributions have indeed one additional parameter, the fundamental frequency $w$ (which is, in general, temperature dependent), fixing its time scale. But this new parameter is easily handled. Take, for instance, the Debye case. The DRF is $w e^{-w t}$ and its LT is $1 /(1+w / s)$. For the Cole-Cole DRF the term $s / w$ is now raised to a power less than one, $(s / w)^{1-\alpha}$. Therefore, defining in the general case

$$
G^{*}\left(s^{\prime}\right)=F^{*}(s), \text { with } s^{\prime}=s / w
$$

by a well known property of the LT, we have that

$$
F(t)=w G(w t)
$$

Nevertheless, in order to avoid the creation of new symbols in the following, the same one will be used for both in Eq.39. That is, we will write $F^{*}\left(s^{\prime}\right)$ for $G^{*}\left(s^{\prime}\right)$, as commonly done. But we have to remember that in order to retrieve the real time behavior the transformation in Eq.40 must be accomplished. We start with the Cole-Cole DRF, generating the corresponding CRF.

\section{V-2.1 The Cole-Cole CRF}

The LT of the Cole-Cole DRF, $\Psi_{c c}^{*}\left(s^{\prime}\right)$, is $[3,18,39,46,48]$

$$
\Psi_{c e}^{*}\left(s^{\prime}\right)=\frac{1}{1+s^{\prime} 1-\alpha}
$$

with $s^{\prime}=s / w$. By Eq.17, as the LT of the corresponding HTDF, it generates for the Cole- Cole HDCRF the very simple result, $\left.F_{C C}(t)\right)$,

$$
F_{C C}(t)=\frac{w(w t)^{-\alpha}}{,(1-\alpha)}
$$

This is the well known Curie-von Schweidler law $[47,23,31]$. It contains two divergencies, one at $t=0$, which was commented upon in the previous section. The other comes from the infinite charge (time integral of the current) it generates for $t \rightarrow \infty$. Different approaches have been proposed to cope with this latter divergence in dielectric theory $[18,36,50]$. In order to improve it in the present conductive case we should rather improve its HTDF (or DRF), which, according to [23], is related to the Mittag-Leffler function. We further add that the Cole-Cole DRF generates, as a HTDF, analytical results for all the values of the exponent $\alpha$ between 0 and 1 when used in the SM treatment of the dispersive photo- current signals. In the original work only the value $1 / 2$ could be fully worked out [9].

\section{V.2-2 The Havriliak-Negami CRF}

The LT of the Havriliak-Negami DRF, [46,48,49], $\Psi_{H N}^{*}\left(s^{\prime}\right)$, is

$$
\Psi_{H N}^{*}\left(s^{\prime}\right)=\frac{1}{\left(1+s^{\prime 1-\alpha}\right)^{\beta}} \text { with } 0 \leq \alpha, \beta \leq 1
$$

In a recent work on aging effects in high density polyethylene, some criticism has been directed at this distribution due to the ability both parameters have of simultaneously influencing the low as well as the high frequency region of the spectrum [48]. This feature appears in the following limiting behavior for the HDCRF, $F_{H N}(t)$,

$$
F_{H N}(t) \approx \frac{w(w t)^{-(1-\beta(1-\alpha))}}{,(\beta(1-\alpha))} \text { for } t \rightarrow 0
$$

and

$$
F_{H N}(t) \approx \frac{w(w t)^{-\alpha}}{\beta,(1-\alpha)} \text { for } t \rightarrow \infty
$$

The point raised in [48] is interesting but perhaps not completely justified according to the following reasoning: Davidson-Cole and Cole-Cole distribution functions result from different deformations imposed upon the simple Debye response, causing, respectively, asymmetry and broadening. The Havriliak-Negami distribution combines those two deformations and it does seem reasonable for the combined deformations to reach both extremes of the frequency spectrum inasmuch as they do that when acting independently.

Returning to our subject, we have obtained by the truncated Widder method of section IV-5, with $k$ not larger than 8 , the primitives $F_{H N}(t)$ for $\alpha=0.25,0.50$, and 0.75 and, in each case, for $\beta=0.25,0.50$ and 0.75 . They are shown in Figs.7,8 and 9, respectively, in a loglog plot. It is seen that while the asymptotic behavior, Eq.45, was already reached for $t^{\prime} \approx 100$, the short time behavior, Eq.44, is not attained for times $\left(t^{\prime}\right)$ of the order of $10^{-2}$. The deviation is higher for smaller $\beta$. 


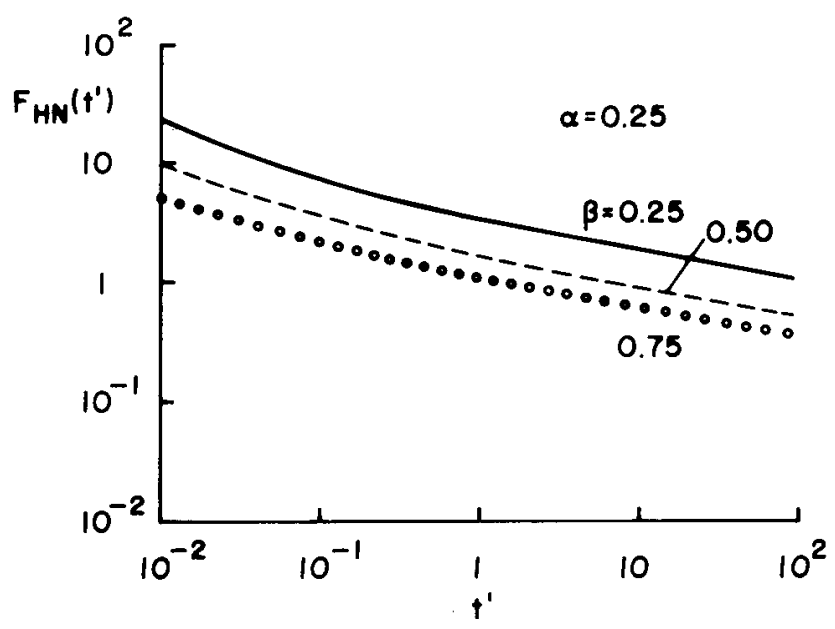

Figure. $7 \log F_{H N} \times \log t^{\prime}$ for $\alpha=0.25$ and $\beta=0.25,0.50$ and 0.75 .

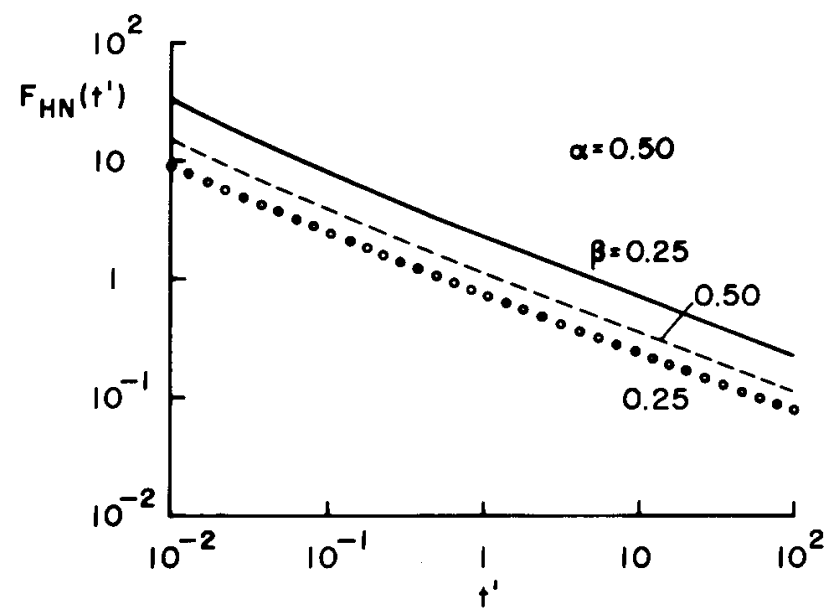

Figure.8 The same as in Fig.7, for $\alpha=0.50$.

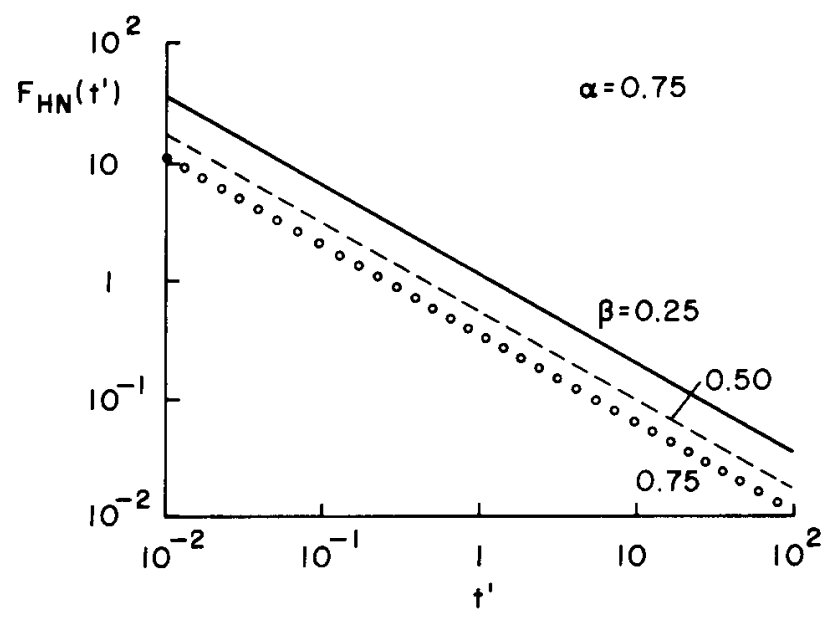

Figure 9. The same as in Figs.7 and 8 but for $\alpha=0.75$.

\section{V.2 Normal Dispersive Responses}

The distinctive character of a NDCRF is to present conductivity. As for the HDCRFs, the LT of the responses, $F^{*}$ and $F_{t}^{*}$, are more easily analyzed with the variable $s^{\prime}=s / w$ and in order to find $F_{t}^{*}$, the long time behavior of $F^{*}$, that is, $\lim _{s^{\prime} \rightarrow 0} s^{\prime} F^{*}$, must be found. Let it be $\underline{a}$. Therefore $F_{t}^{*}\left(s^{\prime}\right)$ is

$$
F_{t}^{*}\left(s^{\prime}\right)=F^{*}\left(s^{\prime}\right)-\frac{a}{s^{\prime}}
$$

Now, in the variable $s$, related to the true time in the LT, there corresponds the limiting behavior aw, which is the value of the true conductivity (not considering the factor $A$ in Eq.10, as already mentioned at the end of Section V-1). Nevertheless $a$ will be called conductivity for short, but the true value will also be mentioned.

\section{V.2-1 The random free energy barrier model}

Macdonald [14] and Dyre [12] have considered the random free energy barrier model for conductive systems (for the dielectric case the model was first proposed by Gevers and Du Pré [53]). In a microscopic description [12] the hopping carrier finds (free) energy barriers of heights varying randomly in space but uniformly in energy from a minimum $U_{\min }$ to a maximum value $U_{\max }$. The probability of a carrier to remain for a time $t$ at a site is given by the function $\Phi(t)$ introduced in Eq.7. Generalizing the two energy barriers treatment in Sec.IV.2, for which $\Phi(t)=a e^{-w_{1} t}+(1-a) e^{-w_{2} t}$, and noting that Eq.28 is the time derivative of this equation, as required by Eq.7, we finally get the following expression for $\Phi(t)$, after performing a change of the integration variable,

$$
\Phi(t)=\frac{1}{\ln \frac{\gamma_{\max }}{\gamma_{\min }}} \int_{\gamma_{\min }}^{\gamma_{\max }} \frac{e^{-\gamma} d \gamma}{\gamma}
$$

where

$$
\gamma_{\max }=\gamma_{0} e^{-U_{\min } / k T} \text { and } \gamma_{\min }=\gamma_{0} e^{-U_{\max } / k T}
$$

and $\gamma_{0}$ is the attempt to escape frequency, common to all barriers, $k$ is the Boltzmann constant and $T$ the absolute temperature. Finding the LT of Eq.47 and then using Eq.8, there results the-Macdonald-Dyre DRF,

$$
\Phi_{D M}^{*}(s)=1-\frac{1}{\ln \frac{\gamma_{\max }}{\gamma_{\min }}} \ln \frac{1+s / \gamma_{\max }}{1+s / \gamma_{\min }}
$$


Note that here the Laplace variable at the moment is $s$ and not $s^{\prime}$ as in the previous section. Dyre [12] makes a further assumption, that the low barriers are in the infrared energy region, while the high ones are of the order of $\mathrm{eV}$ (see however [16]). Therefore $\gamma_{\max }>>\gamma_{\min }$ and $\Psi_{D M}^{*}(s)$ becomes $\Psi_{D}^{*}(s)$ given by

$$
\Psi_{D}^{*}(s)=1-\frac{1}{\lambda} \ln \left(1+\frac{s}{\gamma_{\text {min }}}\right)
$$

with $\lambda=\ln \left(\gamma_{\max } / \gamma_{\min }\right.$. With this simplification Dyre is able to explain regularities observed in ac measurements in the conductive response, to which we now turn our attention. It is found to lead, by Eq.17, to the LT, $\left.F_{D}^{*}\right) s^{\prime}$, now again in a reduced variable $s^{\prime}, s^{\prime}=s / \gamma_{\text {min }}$,

$$
F_{D}^{*}\left(s^{\prime}\right)=\frac{\lambda}{\ln \left(1+s^{\prime}\right)}-1
$$

The conductivity is easily found to be $\lambda$ (or better $\left.\gamma_{\min } \lambda\right)$ and by Eqs.46,40 and 51, the primitive $\gamma_{\min } F_{t D}\left(t^{\prime}\right)$ with $t^{\prime}=\gamma_{\text {min }} t$ may be found. For $\gamma_{\text {max }} \approx$ $1 \mathrm{eV}$ and $\gamma_{\min } \approx .025 \mathrm{eV}$, we have $\lambda=4$. Fig. 10 shows $\log F_{t D}$ as a function of $t^{\prime}$ for $\lambda=2,4$ and 6 . In the present case $\log \Psi_{D}$ is also shown for comparison. A rather subtle behavior is observed between $F_{t D}$ and $\Psi_{D}$ concerning the variation of $\lambda$ : for $\lambda=2, \Psi_{D}$ is higher than $F_{t D}$; for $\lambda=4$ the same trend remains for short times, but for longer times the differences almost disappear. Finally, for $\lambda=6, \Psi_{D}$ becomes smaller than $F_{t D}$ over the whole time scale.

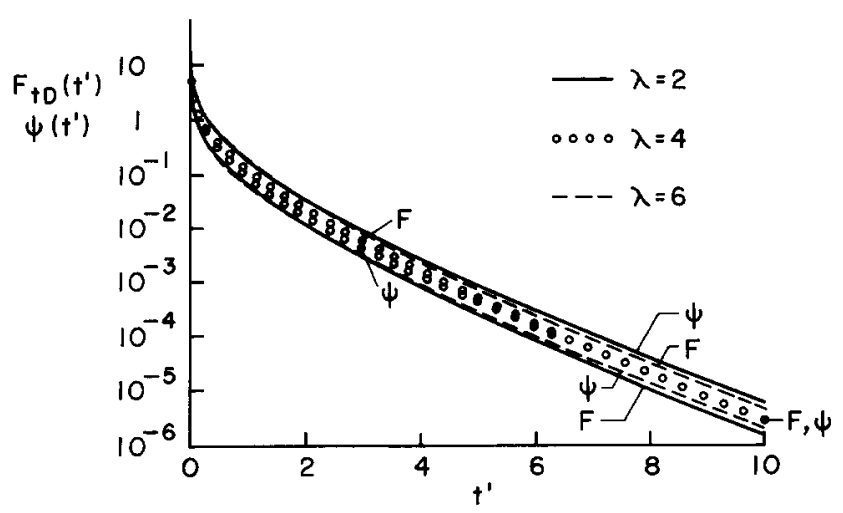

Figure 10. The Dyre random free energy barrier model, log $F_{t D} \times t^{\prime}$ and $\log F_{t D} \times t^{\prime}$ and $\log \psi_{D} \times \log t^{\prime}$ for $\lambda=2,4$, and 6. $F_{t D}$ is smaller than $\psi_{D}$ for $\lambda=2$ and 4 , although already almost equal to it at large times; for $\lambda=6, F_{t D}$ becomes greater than $\psi_{D}$.

One of the referees called our attention aboutrecent developments aimed at improving the random free energy model $[49,50]$.

\section{V.2-2 The Davidson-Cole CRF}

The LT of the Davidson-Cole DRF, $\Psi_{D C}^{*}\left(s^{\prime}\right)$, is $[3,19,24,39,46,48,52,54]$

$$
\Psi_{D C}^{*}\left(s^{\prime}\right)=\frac{1}{\left(1+s^{\prime}\right)^{\beta}}
$$

with $s^{\prime}=s / w$. Its $\mathrm{CRF}, F_{D C}^{*}\left(s^{\prime}\right)$, is

$$
F_{D C}^{*}\left(s^{\prime}\right)=\frac{1}{\left(1+s^{\prime}\right)^{\beta}-1}
$$

and it is found to lead to a non-zero conductivity $1 / \beta$ (the real one is $w / \beta$ ). By Eq.46 we get the LT of its decaying component, $F_{t D C}^{*}\left(s^{\prime}\right)$. For short times $F_{t D C}\left(t^{\prime}\right)$ behaves as

$$
F_{t D C}(t) \approx \frac{w t^{\prime \beta-1}}{,(\beta)}, \quad t^{\prime}=w t
$$

which is close to the exact DRF, $\Psi_{D C}(t)$, for short times which is

$$
\Psi_{D C}(t)=\frac{w t^{\prime} \beta-1}{,(\beta)}, \quad t^{\prime t^{\prime}}=w t
$$

In Fig.11 we plot $\log F_{t D C}$ as a function of $t^{\prime}$ after an inversion by the truncated Widder method for $\beta=0.25,0.50$ and 0.75 . An exponential like decay, independent of the $\beta$ value, is observed for long times.

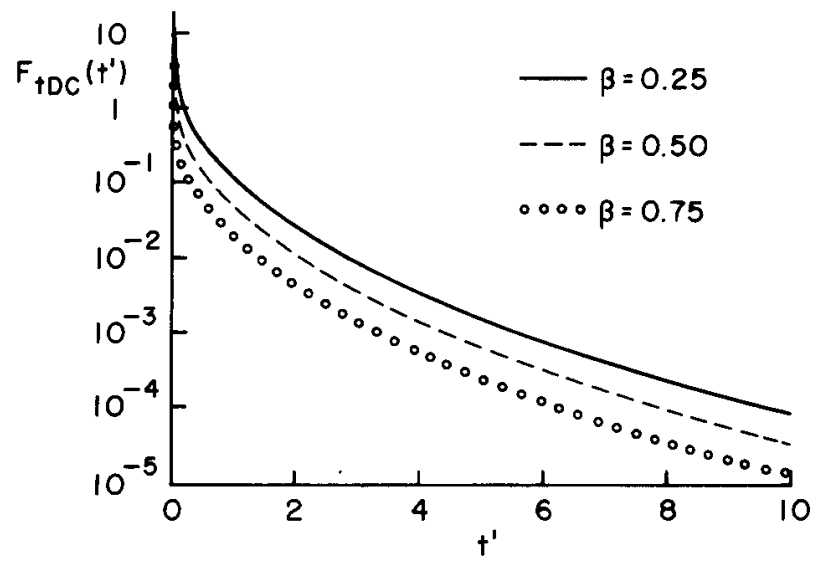

Figure 11. The Davidson-Cole NCRF, $F_{t D C}$, in a plot of $\log F_{t D C}$ as a function of $t^{\prime}$.

\section{V.2-3 The one- half- stretched- exponential}

The Kohlrausch-Williams-Watts stretched exponential $[1,37,52,54,55]$ corresponds to the function $\Phi(t)$, defined in Eqs. 7 and 8, that is, the probability the carrier has of staying at the same site for a time $t$ after arrival, 


$$
\Phi(t)=e^{-(w t)^{\beta}}
$$

We know that in order to have quick access to the CRFs we need the LT of $\Psi(t)$, the negative of the time derivative of $\Phi(t)$. The only available LT of $\Phi(t)$ in Eq.56 is for $\beta=1 / 2$ and now we stick to this particular case. The CRF, $F_{K W W}^{*}\left(s^{\prime}\right)$, with $s^{\prime}=s / w$, is found to be

$$
F_{K W W}^{*}\left(s^{\prime}\right)=\frac{\sqrt{\pi / 2 s^{\prime}} e^{1 / 4 s^{\prime}} \operatorname{Erfc}\left(1 / 2 \sqrt{s^{\prime}}\right.}{1-\sqrt{\pi / 4 s^{\prime}} e^{1 / 4 s^{\prime}} \operatorname{Erfc}\left(1 / 2 \sqrt{s^{\prime}}\right)}
$$

where $\operatorname{Erfc}(z)$ is the complementary error function of the argument $z$ [42]. The conductivity is $1 / 2$ (the true one is $w / 2)$ and the decaying part of $F_{K W W}\left(t^{\prime}\right)$, $F_{t K W W}\left(t^{\prime}\right)$, is then found. Fig.12 shows $\log F_{t K W W}$ as a function of $\sqrt{t^{\prime}}$ obtained by the truncated Widder method with $k=6$ and 8 . Some instability occurs for $\sqrt{t^{\prime}} \approx 10$, that is, $t^{\prime} \approx 100$. Anyway an $e^{-\sqrt{t^{\prime}}}$ behavior holds for sufficiently long times.

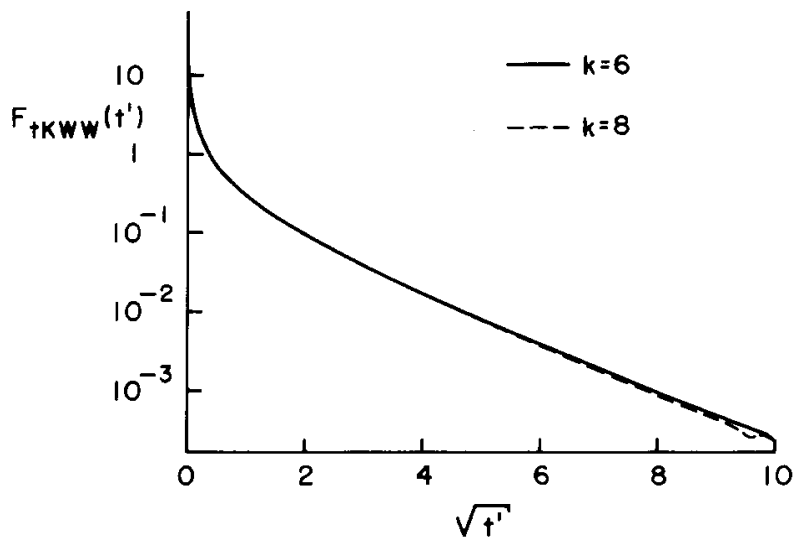

Figure 12. The NCRF $1 / 2$ stretched exponential decay, log $F_{t K W W}$ as a function of $\sqrt{t^{\prime}}$, for $k=6$ and 8 . Instability in the deconvoluted curve is observed for high values of $\sqrt{t^{\prime}}$, $t^{\prime} \approx 100$.

Extensive analysis of previous work and new results on the stretched exponential response appear in [53].

\section{V.2-4 Master Curves}

We have seen in Eq.40 that in the cases considered here, $F(t)$ may be put in the following form of

$$
F(t)=w G(w t)
$$

where $w$ becomes $\gamma_{\text {min }}$ for the Dyre CRF of Section V$2-1$. As already mentioned $w$ is usually a function of the temperature and therefore Eq.54 becomes an instance of the time- temperature superposition principle [55]. As is well known, a master curve may be drawn from a set of functions after appropriated re-scaling of the quantities involved $[39,57,58,59]$. From the dielectric theory, it is known that Eq.54 results from a distribution of relaxation times with a single activation energy. The present analysis dealt with non-Debye conductive responses leading however to the same general result.

\section{Concluding Remarks}

The existence of a structured ac response in disordered materials is a well known fact [12,13,22]. Therefore a time dependent conductivity is expected to appear when a step voltage is applied to them in planar symmetry. In Dyre's approach [12] the time dependent as well as the stationary conductivity are derived from a single function in a quite consistent scheme so that reasonable results come out of the calculations for well behaved HTDFs (decreasing functions of time). A behavior similar to that observed in a poled dielectric material is predicted, including return currents after a short. But while simple explanations may be advanced for time dependent currents in dielectric systems, they are far less intuitive in the conductive case. In the former we say that the populations of dipoles pointing in the same and in the opposite direction as that of the electric field are perturbed and the observed currents arise from the changing dipole configuration. In the conductive case dipoles are formed by the motion of the mobile charge with respect to its fixed partner. But, the existence of conductive return currents of long duration, as usually found in the dielectric case, seems to go against our intuition and therefore, on this basis, we expect them to decay faster than the dipolar ones. Of course the same would be true for the corresponding conductive absorption current. For the phenomenological approach $[14,16,17,11]$ the conductive system is considered as a series distribution of differential resistors and capacitors in parallel. The return current is easily explained and its energetics understood by the inhomogeneous charging of the system during poling.

From the experimental point of view, a thorough analysis of conduction in the planar symmetry requires the knowledge of the role played by the electrodes and their charge exchange with the sample. These processes might create space-charge layers near the electrodes, perturbing the bulk current. AC experiments do not present such difficulty.

Another point concerns thin samples. The measured external current of a conductive system provides 
a mean value of the property inside the sample. But as noticed in [60] truly random systems require infinite samples and therefore we expect somewhat nonreproducible results when dealing with especially thin samples. Quantitative analysis is missing.

We have seen in sub-section $\mathrm{V}-2$ and subsequent ones that the distributions are defined in terms of a frequency which is, in general, temperature dependent. Therefore a common dependence on temperature of the time dependent and as well as of the steady state current might be an indication that the system under study presents a conductive rather than a dielectric behavior. Some glasses belong to this class [61,12]. A similar behavior was found in $\alpha$-PVDF [62], although in this case the return current was of long duration and, therefore, might not be of the conductive type, according to what has been said before.

\section{Acknowledgments}

The authors are indebted to Prof. Gross for reading and for the many suggestions made, all of them now incorporated into the text, as well as, to Prof. Mariangela T. Figueiredo for the permanent assistance she gave in computational problems. The authors thank $\mathrm{CNPq}$ and CAPES for the research grant (GFLF) and the doctorate fellowship (SCC), respectively. It should also be mentioned that the present final version is strongly indebted to the criticism of the referees and to Prof. Osvaldo N. Oliveira Jr. who kindly revised the text.

\section{References}

1. B. Gross, IEEE Trans. Electr. Insul. EI,-21, 249 (1986).

2. B. Gross, J. Chem. Phys. 17, 866 (1949).

3. B. Hilczer and J. Malecki, Electrets, PWn-Polish Sci. Publ. Warsaw, and Elsevier, Sci. Publ., Amsterdam (1986).

4. G.M. Sessler, Ed., Topics in Applied Physics, vol. 33, Electrets, 2nd Edit., Springer- Verlag (1987), specially last chapter by R. Gerhard-Multihaupt, B. Gross and G.M. Sessler.

5. J.R. Macdonald and C.A. Barlow, Jr, Rev. Modern Phys. 35, 940 (1963).

6. B. Gross, An. Acad. Bras. Cienc. 12, 203 (1940).

7. V.M. Krenke, E.W. Montroll and M.F. Shlesinger, J. Stat. Phys. 9, 45 (1973).

8. H. Scher and M. Lax, Phys. Rev. B 7, 4491 (1973).

9. E.W. Montroll and H. Scher, J. Stat. Phys. 9, 101 (1973).

10. H. Scher and E.W. Montroll, Phys. Rev. B 12, 2455 (1975).
11. G.F. Leal Ferreira and L.E. Carrano de Almeida, Phys. Rev. B 19,6601 (1979).

12. J.C. Dyre, J. Appl. Phys. 64, 2456 (1988).

13. G.A. Niklasson, J. Appl. Phys. 66, 4350 (1989).

14. J. Ross Macdonald, J. Appl. Phys. 58, 1955 (1985).

15. J. Ross Macdonald, J. Appl. Phys. 62, 62, R51 (1987).

16. J. Ross Macdonald, J. Appl. Phys. 65, 4845 (1989).

17. J.A. McInnes, P.N. Butcher and J.D. Clark, Philos. Magaz. B 41, 1 (1980).

18. K. Jonscher, Dielectric Relaxation in Solids, Chelsea Dielectric, London (1983).

19. J.R. Macdonald, Ed., Impedance SpectroscopyEmphasizing Solid Materials, Wiley-Interscience, N.York (1987).

20. G.F. Leal Ferreira and L.E. Carrano de Almeida, Phys. Rev. B 56, 11579 (1997).

21. A. Dekker, Solid State Theory, Prentice Hall Inc., Englewood (1962), Ch.7.

22. A. Hunt, J. Non-Cryst. Sol. 144, 21 (1992).

23. B. Gross, J. Appl. Phys. 18, 212 (1947).

24. B. Gross and H. Pelzer, J. Appl. Phys. 22, 1035 (1951).

25. J.A. Giacometti and O.N. Oliveira Jr., IEEE Trans. Electr. Insul. EI-27, 924 (1992).

26. B. Gross, J.A. Giacometti and G.F. Leal Ferreira, CEIDP Ann. Report, 39-44 (1981).

27. J.A. Giacometti, P.A. Ribeiro, M. Raposo, J.N. Marat-Mendes and A.S. DeReggi, J. Appl. Phys. 78, 5597 (1995).

28. B. Gross, Philos. Magaz. 51, 543 (1950).

29. B. Gross, Mathematical Structure of the Theories of Viscoelasticity, Hermann \& Cie., Paris (1953).

30. H. Silva and B. Gross, Phys. Rev. 60, 684 (1941).

31. B. Gross and H. Pelzer, Proc. Royal Soc. A 210, 434 (1951).

32. B. Gross, Zeitsch. Naturf. 6a, 676 (1951).

33. B. Gross, J. Appl. Phys. 62, 2763 (1987).

34. G.F. Leal Ferreira and B. Gross, J. Appl. Phys. 68, 2526, (1990).

35. A.K. Jonscher, J. Electrost. 3, 53 (1977).

36. L.A. Dissado and R.M. Hill, J. Chem. So. Faraday Trans. 2 80, 291 (1984).

37. K.L. Ngai, R.W. Rendell, A.K. Rajagopal and S. Teitler, Ann. N. Y. Acad. Sci. 484, 150 (1986).

38. K.L. Ngai, A.K. Rajagopal, R.W. Rendell and S. Teitler, IEEE Trans. Electr. Insul. EI- 21, 313 (1986).

39. J. van Turnhout, Thermally Stimulated Discharge of Polymer Electrets, Elsevier Sci. Publ., Amsterdam (1975).

40. G. Williams and D.C. Watts, IEEE Trans. Electr. Insul. EI-20, 843 (1985). 
41. The same as Ref.7, especially Eqs. A9 and A10. Note that a factor is missing inside the bracket in Eq.A9.

42. M. Abramowitz and I.A. Stegun, Ed.Handbook of Mathematical Functions, Dover Publ. Inc., N.York (1965).

43. G.E. Roberts and H. Kaufman, Table of Laplace Transforms, W.B. Saunders Co., Philadelphia (1966).

44. F. Oberhettinger and L. Badii, Tables of Laplace Transform, Springer-Verlag, Berlin, (1973).

45. D.V. Widder, The Laplace Transform, Princeton Univ. Press, Princeton (1946), Ch.VII.

46. C.J.F. Bottcher and P. Bordewijk, The Theory of Electrical Polarization, Vol.II, Elsevier Sci. Publ. Amsterdam (1978)

47. B. Gross, J. Appl. Phys. 19, 257 (1948).

48. D.K. Das-Gupta and P.C.N. Scarpa, IEEE Trans. Diel. Elect. Insul. 3, 366 (1996).

49. J.C. Dyre, Phys. Rev. B 47, 9128 (1993).

50. J.R. Macdonald, Phys. Rev. B49 II, 9428 (1994).

51. S. Yagihara, R. Nozaki, S. Takeishi and S. Mashimo, J. Chem. Phys. 79, 2419 (1983).

52. B. Gross, J. Appl. Phys. 67, 6399 (1990).

53. M. Gevers and F.K. Du Pré, Trans. Faraday Soc.
42A, 47 (1946).

54. J.R. Macdonald, J. Non-Crystal. Solids 197, 83 (1996).

55. J.R. Macdonald, J. Non-Crystal. Solids 204, 309 (1996).

56. C.P. Lindsey and G.D. Patterson, J. Chem. Phys. 73, 3348 (1980).

57. B. Gross, J. Appl. Phys. 40, 3397 (1969).

58. B. Gross, Curso sobre Teoria de Relaxação Dielétrica e Tópicos sobre a Teoria da Resposta Linear (A Course on the Theory of Dielectric Relaxation and Topics on the Linear Response Theory), Notes, Vol.III, IFQSC-USP (1981).

59. J. Ross Macdonald, J. Chem. Phys. 36, 345 (1962).

60. P.N. Butcher, Philos. Magaz. B42, 799 (1980).

61. J.M. Stevels, Electrical Properties of Glass, Handbuch der Physik, Ed. S.Flugge, Band XX, Springer-Verlag, Berlin (1957).

62. H.A. Aquino, Experiências de polarização $e$ condução nos polímeros PVDF e FEP (Polarization and conduction experiments in the PVDV and FEP polymers), Master Dissertation, IFQSCUSP (1983). 